\title{
Cancer occurrence in patients with inflammatory bowel disease in treatment with anti-TNF: report of three cases and literature review
}

\section{Ocorrência de câncer em pacientes com doença inflamatória intestinal em tratamento} com anti-TNF: relato de três casos e revisão da literatura

Lucas Camargo Gamba Martins do Amaral ${ }^{1}{ }^{\oplus}$, Luiz Henrique Locks Correa ${ }^{2}$, Cassiano Coral Accord ${ }^{2}$, Thamy dos Santos' ${ }^{2}$, Beatriz de Oliveira Kock², Kaiser de Souza Kock ${ }^{1}$

\begin{abstract}
Case report of three patients with inflammatory bowel disease who underwent treatment with biology therapy and developed respectively: non-Hodgkins lymphoma, colorectal adenocarcinoma, and cholangiocarcinoma after long-term follow-up. They demonstrated that data are currently inconclusive about the development of long-term anti-TNF neoplasias.
\end{abstract}

Keywords: Inflammatory bowel diseases; Tumor necrosis factors; Lymphoma, NonHodgkin; Cholangiocarcinoma.

\begin{abstract}
RESUMO
Relato de caso de três pacientes com doença inflamatória intestinal que realizaram tratamento com terapia biológica e desenvolveram, respectivamente: linfoma nãoHodgkin, adenocarcinoma colorretal e colangiocarcinoma, após acompanhamento a longo prazo. Eles demonstraram que os dados atualmente são inconclusivos sobre o desenvolvimento de neoplasias anti-TNF a longo prazo.
\end{abstract}

Descritores: Doenças inflamatórias intestinais; Fatores de necrose tumoral; Linfoma não-Hodgkin; Colangiocarcinoma.

1. Unisul - Universidade do Sul de SC, Medicina - Tubarão - Santa Catarina.

2. Hospital Nossa Senhora da Conceição, Serviço de Cirurgia - Tubarão - Santa Catarina - Brazil.

Financial support: none to declare.

Conflicts of interest: The authors declare no conflict of interest relevant to this manuscript.

Correspondence author: Lucas Camargo Gamba Martins do Amaral.

E-mail: hadtobcm@gmail.com 


\section{INTRODUCTION}

Inflammatory bowel disease (IBD) is a chronic immune-mediated disease with a recurring course that mainly affects the gastrointestinal tract, primarily with chronic inflammation in the intestine. ${ }^{[1]}$ Crohn's disease (CD) and ulcerative colitis (UC) are the two main diseases in this definition. CD is characterized by transmural inflammation, which may involve the entire gastrointestinal tract from the mouth to the perianal area discontinuously, but the ileum and colon are preferentially affected. ${ }^{[1]}$ In UC, continuous mucosal inflammation of the colon can be observed, affecting a variable extent of the continuous range of the rectum and colon. ${ }^{[1]}$

Pharmacological therapies have been employed for its remission, and among them, biological therapy stands out, and its main class of drug being the tumor necrosis anti-factor (anti-TNF). ${ }^{[1]}$ With its primary function being mucosal repair, treatment response can reach up to $69 \%$ of patients with CD and $70 \%$ with UC under continuous infliximab treatment. ${ }^{[2]}$ Up until now, the occurrence of opportunistic infections and sometimes malignancies has been evidenced as a consequence of its long-term use, ${ }^{[2]}$ and, among these malignancies, the most reported are lymphomas and solid tumors of the digestive tract. ${ }^{[3]}$

IBD is often associated with extraintestinal manifestations, such as arthritis, ocular involvement, skin diseases, primary sclerosing cholangitis (PSC), venous or arterial thromboembolism or pulmonary involvement, or concomitant immune-mediated diseases. ${ }^{[1]}$ This study presents three case reports of patients with inflammatory bowel disease that underwent treatment with biological therapy, and, at some point, developed neoplasias.

\section{CASE REPORT}

\section{Case 1}

GK, male, 60-years-old, with onset of rectal bleeding and diarrhea in 2009, being diagnosed by colonoscopy with left colitis compatible with UC, and confirmed by histopathological examination. He initially used mesalazine and prednisone, showing, in the first year, a corticosteroid-dependent disease. He was started with azathioprine at a dose of 150mg/ day with partial control and occasional relapses, requiring a corticosteroid pulse. Relapses became more frequent, and 3 years after the onset of the disease, the biological therapy with adalimumab was introduced. The disease remained partially controlled until March 2018, when he developed an anal abscess, which was uneventfully drained. After this date, he presented recurrences of tenesmus, rectal bleeding, diarrhea, and weight loss of $7 \mathrm{~kg}$ (15.4lbs) in 3 months. A colonoscopy evidenced an intense mucosal alteration in the rectosigmoid region, with an extension of approximately $12 \mathrm{~cm}$ (4.7in), hardened and with intense friability, ulcerations, and adhered fibrin. The main hypothesis was neoplastic degeneration, but the biopsies were negative for malignancy. Based on the long-term evolution of the disease, clinical and endoscopic findings, the patient underwent surgical treatment involving abdominal rectosigmoidectomy by videolaparoscopy with primary colorectal anastomosis. The cavity inventory showed only the rectosigmoidal thickened area. Histopathological examination showed morphological findings suggestive of large cell nonHodgkin's lymphoma (Figures 1 and 2).

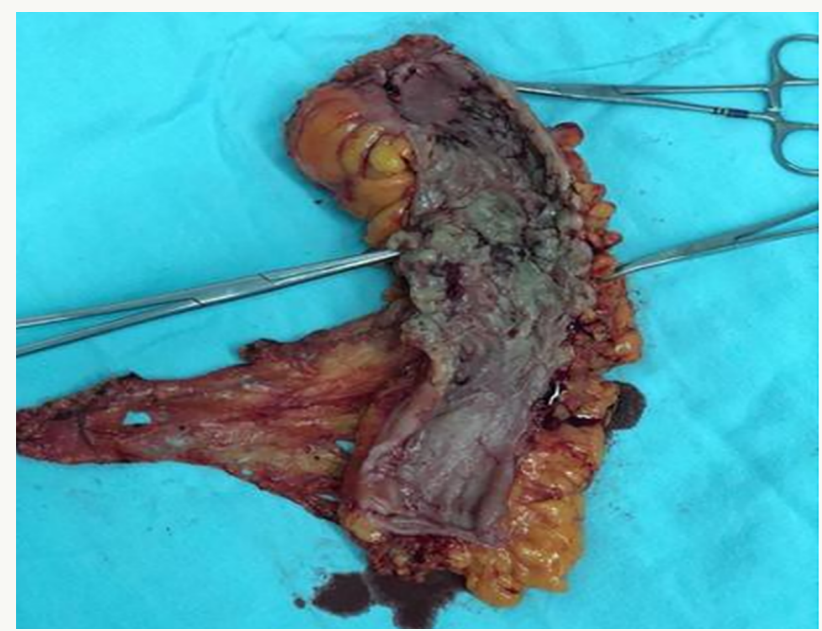

Figure 1. Rectosigmoid colon with morphological findings suggestive of large cell non-Hodgkin's lymphoma.

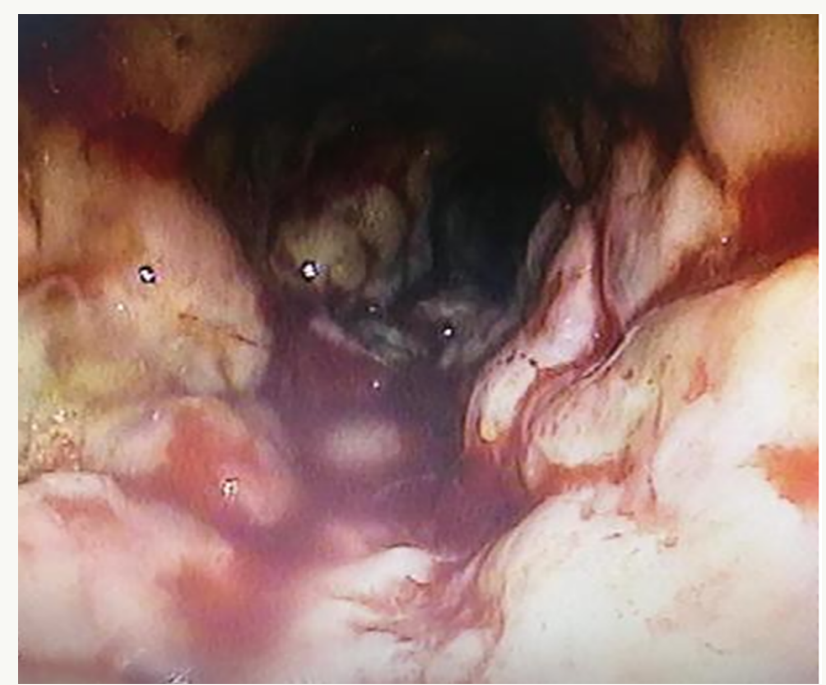

Figure 2. Evidence of mucosal alteration in the rectosigmoid region, with an extension of approximately $12 \mathrm{~cm}$ (4.7in), hardened, with intense friability, ulcerations, and adhered fibrin.

\section{Case 2}

VG, 67-years-old, male, initially developed tenesmus and rectal bleeding 15 years ago (2004). He underwent colonoscopy showing intense proctitis, compatible with UC. He started mesalazine and corticosteroid therapy with partial symptom control. After being oligosymptomatic for a year, he developed erythema nodosum and complex anorectal fistula. Immunosuppressive therapy with azathioprine was started, and the possibility of anorectal Crohn's 
disease was evaluated. The colonoscopy did not show other inflammatory areas in the colon or terminal ileum. There was then a progressive worsening of the anorectal disease, with the appearance of new fistulas and the development of pyoderma gangrenosum. He received a corticosteroid pulse and the biological therapy with infliximab was initiated (2008). There was a control of anorectal manifestations with superficial abscess drainage, setons placement, and maintenance of biological and immunosuppressive therapy for about 4 years, and the setons were progressively removed during this period. The colonoscopy at the time showed mild to moderate proctitis. After 3 years, the patient returned with the onset of intense tenesmus, mucorrhea, and rectal bleeding. A colonoscopy was performed, showing an extensive polypoid lesion, occupying the entire rectum, with intense friability. The biopsies showed high-grade adenomas with foci of adenocarcinoma. Based on clinical evolution and histopathological diagnosis, abdominoperineal rectal amputation by video laparoscopy was performed and the pathology of the surgical specimen was demonstrated (Figure 3).

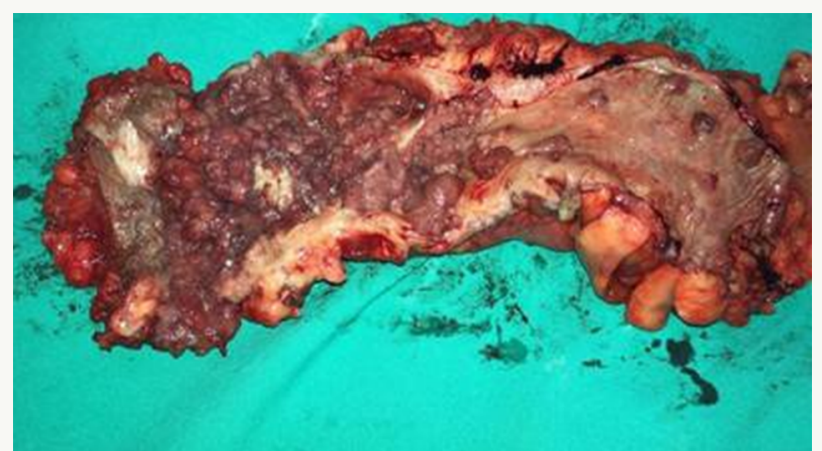

Figure 3. Abdominoperineal rectal amputation with polypoid lesions, occupying the entire rectum, with intense friability.

\section{Case 3}

MS, 68-years-old, male, diagnosed with anorectal Crohn's disease 11 years ago (2008). He was initially medicated with corticosteroids, and mesalazine in combination with azathioprine. After 2 years of evolution, and with recurrent symptoms, he began to use infliximab, and with withdrawal of corticosteroids and mesalazine, but maintaining azathioprine. After a year of use of biological therapy, he then developed adverse reaction to infliximab, presenting severe arthralgias and skin rash, with the infliximab being then replaced by adalimumab. There was an initial progression of perineal disease, with the appearance of complex fistulas, which required drainage and setons placement, showing progressive improvement. Subcutaneous fistulectomies were performed and setons were removed after a year, with subsequent closure of the fistulas, leaving only perineal sequelae. The disease progressed satisfactorily over the following few years, but, 5 months ago, he began to show inappetence, anorexia, and progressive weight

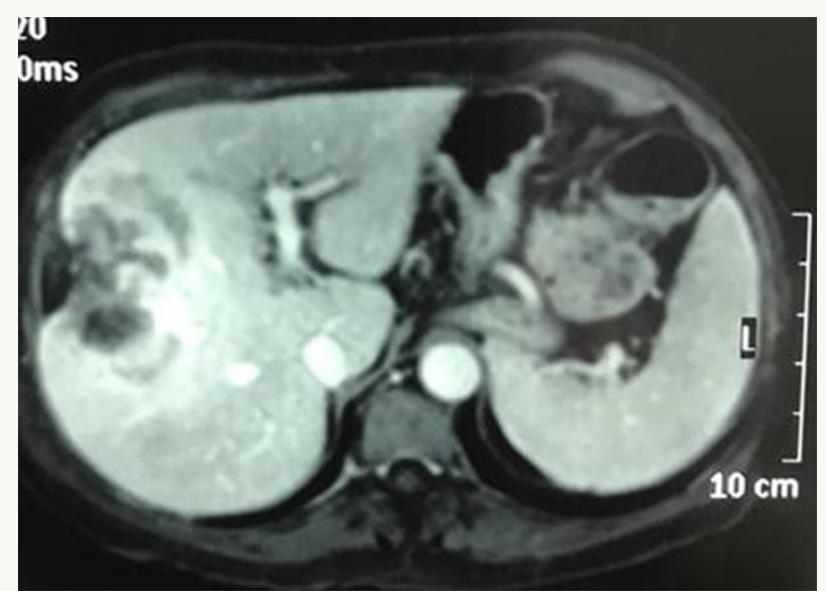

Figure 4. Tumoral lesion in the hepatic segments VIII, V, and IV; and areas of necrosis compatible with cholangiocarcinoma.

loss. A new colonoscopy was performed, showing no active inflammatory bowel disease. A new abdominal magnetic resonance showed a tumoral lesion in the hepatic segments VIII, V, and IV; and areas of necrosis compatible with cholangiocarcinoma (Figure 4).

\section{DISCUSSION}

The pathogenesis of IBD is still unclear, but a new hypothesis suggests that the inflammation in IBD is a result of dysregulation of the mucosal immune response to commensal flora antigens in genetically susceptible hosts. This means that, despite the genetic susceptibility of this disease, a variety of environmental factors can trigger its clinical manifestations. The goal of medicine is to change the course of the disease by achieving mucosal healing.

Drugs used to treat IBD include 5-aminosalicylic acid (5-ASA), immunomodulators (IMM) and biological agents. 5-ASA is an intestine-specific aminosalicylate drug that acts locally in the intestine and is used for induction and maintenance treatment of UC patients. $[2,4]$ Since it is rapidly absorbed in the jejunum, only $20 \%$ of it reaches the terminal ileum and colon; because of that, a variety of 5-ASA compounds have been developed to prevent 5-ASA from being absorbed in the proximal end of the gastrointestinal tract and, thus, increasing delivery to the colon. IMM, including azathioprine, 6-mercaptopurine and methotrexate, has shown its efficacy, mainly as a maintenance therapy for IBD remission. ${ }^{[2,4]}$

Azathioprine is a derivative of the purine-simulated antimetabolite thioguanine. It is a prodrug that is transformed into its main metabolite 6-mercaptopurine by non-enzymatic nucleophilic attack of thiol- containing compounds (such as glutathione) present in red blood cells and other tissues. 6-mercaptopurine is then metabolized in the liver and intestine by one of three enzymes. Methotrexate is a folic acid analog that inhibits the synthesis of purines and pyrimidines, which explains its efficacy in cancer treatment, and its adenosine-mediated anti-inflammatory effect makes 
it a good substitute for immunosuppressive drugs. Methotrexate is a prodrug that is active only when the cells are polyglutamic.

In biological agents, anti-tumor necrosis factor TNF-a drugs have been widely used in the treatment of IBD. ${ }^{[5,6]}$ TNF-a plays a fundamental role in the immune response, having been associated with carcinogenesis when suppressed, or contributing to inflammatory diseases due to its dysfunction. ${ }^{[3]}$ AntiTNF-a is a monoclonal antibody that blocks the proinflammatory cytokine TNF-a associated with the pathogenesis of IBD.

In Europe, the approved anti-TNF-a drugs are infliximab (a mouse-human chimeric IgG1 antibody), adalimumab (a fully human recombinant IgG1 antibody) and golimumab (A human IgG1 anti-TNF-a). All were approved for induction and maintenance therapy. Anti-integrin agents natalizumab (humanized IgG4 antibody against a4 integrin) and vedolizumab (humanized IgG1 antibody against a4 $\beta 7$ integrin) regulate the intestinal lymphocyte transport, and uteuzumab is a monoclonal antibody that blocks interleukin IL-12 and IL-23. ${ }^{[5,6]}$

Inflammatory bowel disease has been singly identified as an important risk factor for the development of lymphoma, bile duct and intestinal cancer. ${ }^{[7]}$ Regarding the development of lymphomas, it shows no significant changes when treated with anti-TNF alone, but presents an increased relative risk in combination therapy with immunomodulators 4.4 (95\% Cl:3.4-5.4). ${ }^{[8]}$ Although there are reported cases of tumor development, Biancone et al. (YEAR) ${ }^{x}$, followed 404 patients with IBD over a 5-year period using infliximab and found no increase in malignancy compared to the control group, and a similar result was obtained with Lichtenstein et al. (YEAR) ${ }^{x}$, in a study with 6,273 patients.

Some studies show a relative increase of appearance of colorectal adenocarcinoma and cholangiocarcinoma in patients treated with infliximab for other inflammatory diseases such as Wegener's granulomatosis and rheumatoid arthritis. ${ }^{[8]}$ The risk of developing progressive multifocal leukoencephalopathy associated with John Cunningham virus in patients receiving natalizumab limits its use. Vedolizumab and ustekinumab have shown beneficial effects on IBD patients in phase III studies, respectively. The main long-term complications of IBD immunosuppressive therapy are opportunistic infections and the risk of cancer development. Several mechanisms have been proposed to promote carcinogenesis, including changes in DNA, activation of oncogenes, reduction in physiological and immune surveillance, and promotion of the role of oncogenic viruses. [10] Anti-TNF-a agents have a dual effect on tumor progression, because TNF-a can show anti-tumor effects by initiating apoptosis of malignant cells, but the secretion of most tumors can also help cell survival and increase tumor proliferation. ${ }^{[11]}$
Another case report found that two patients in use of anti-TNF-a developed B- and T-cell lymphoma in a short time of treatment. ${ }^{[12]}$ There are some studies showing a correlation between lymphoma and IBD without the use of anti-TNF-a, and a correlation with the disease time and risk factors of the patients, suggesting that treatment does not appear to be correlated. ${ }^{[13]}$ In order to understand the relationship between IBD and cancer, IBD patients should consider three different situations: 1) cancer associated with local chronic inflammation (IBD-related cancer); 2) cancer associated with the use of immunosuppressive drugs in this population (treatment-related cancer associated with immunosuppression); and 3) any unrelated cancer that may occur in IBD patients. ${ }^{[14]}$ Thus, the risk for developing cancer with anti-TNF-a treatment has been found controversial or often not having significant data, being considered a potent drug to prevent IBD progression, promoting mucosal healing and preventing the development of colitis-related cancer. It is considered potentially safe for shortterm use and periodic examinations are indicated to prevent complications. Further evaluation with longterm biological therapy requires further study.

\section{REFERENCES}

1. Cardozo WS, Sobrado CW. Doença inflamatória intestinal. $2^{\text {a }}$ ed. Barueri: Manole; 2014. v. 2.

2. Dignass A, Lindsay JO, Sturm A, Windsor A, Colombel JF, Allez M, et al. Second European evidence- based consensus on the diagnosis and management of ulcerative colitis. Part 2: current management. J Crohns Colitis. 2012 Dec;6(10):991-1030.

3. Raval G, Mehta P. TNF-a inhibitors: are they carcinogenic?. Drug Healthc Patient Saf. 2010;2:241-7.

4. Dignass A, Van Assche G, Lindsay JO, Lémann M, Söderholm J, Colombel JF, et al. The second European evidence-based consensus on the diagnosis and management of Crohn's disease: current management. J Crohns Colitis. 2010 Feb;4(1):28-62.

5. Sandborn WJ, Feagan BG, Marano C, Zhang $H$, Strauss R, Johanns J, et al. Subcutaneous golimumab maintains clinical response in patients with moderate-to-severe ulcerative colitis. Gastroenterology. 2014 Jan;146(1):96-109.e1.

6. Sandborn WJ, Gasink C, Gao LL, Blank M, Johanns J, Guzzo C, et al. Ustekinumab induction and maintenance therapy in refractory Crohn's disease. N Engl J Med. 2012 Oct;367(16):1519-28.

7. Pedersen N, Duricova D, Elkjaer M, Gamborg M, Munkholm P, Jess T. Risk of extra-intestinal cancer in inflammatory bowel disease: metaanalysis of population-based cohort studies. Am J Gastroenterol. 2010 Jul;105(7):1480-7. 
8. Sultan K, Shapira J. Inflammatory bowel disease and lymphoma: a comprehensive review for the general gastroenterologist. Pract Gastroenterol. 2012 Aug;13.

9. Cohen RB, Dittrich KA. Anti-TNF therapy and malignancy-a critical review. Can J Gastroenterol Hepatol. 2001 Jun;15(6):376-84.

10. O'Donovan $\mathrm{P}$, Perrett $\mathrm{CM}$, Zhang $\mathrm{X}$, Montaner B, $\mathrm{Xu} Y Z$, Hardwood C, et al. Azathioprine and UVA light generate mutagenic oxidative DNA damage. Science. 2005 Sep;309(5742):1871-4.

11. Beaugerie L. Inflammatory bowel disease therapies and cancer risk: where are we and where are we going?. Gut. 2012 Apr;61(4):476-83.
12. Molnár T, Farkas K, Nagy F, Szepes Z, Wittmann T. Lymphomas in two IBD patients treated with anti- TNF-a mono or combination therapy: is hepatosplenic lymphoma really the "old maid"? Inflamm Bowel Dis. 2011 Sep;17(9):2025-6.

13. Mourabet ME, Hashash JG, Sun NH, Issa M, Katz JA, Regueiro M, et al. Clinical course of Crohn's disease following treatment of lymphoma. Inflamm Bowel Dis. 2011 Jun;17(6):1265-9.

14. Jess T, Gamborg M, Matzen P, Munkholm P, Sørensen TI. Increased risk of intestinal cancer in Crohn's disease: a meta-analysis of populationbased cohort studies. Am J Gastroenterol. 2005 Dec;100(2):2724-9. 